Short communication

\title{
Nonverbal behaviors are associated with increased vagal activity in major depressive disorder: Implications for the polyvagal theory
}

\author{
Raquel A. Fernandes ${ }^{\mathrm{a}}$, Juliana T. Fiquer ${ }^{\mathrm{b}}$, Clarice Gorenstein ${ }^{\mathrm{b}, \mathrm{c}}$, Lais Boralli Razza ${ }^{\mathrm{a}}$, \\ Renério Fraguas $\mathrm{Jr}^{\mathrm{a}, \mathrm{d}}$, Lucas Borrione ${ }^{\mathrm{a}, \mathrm{d}}$, Isabela M. Benseñor ${ }^{\mathrm{a}}$, Paulo A. Lotufo ${ }^{\mathrm{a}}$, \\ Eduardo Miranda Dantas ${ }^{\mathrm{e}}$, Andre F. Carvalho ${ }^{\mathrm{f}}$, André R. Brunoni ${ }^{\mathrm{a}, \mathrm{d}, \mathrm{g}, *}$

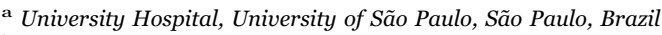 \\ b LIM-23, Institute of Psychiatry, University of São Paulo, São Paulo, Brazil \\ c Department of Pharmacology, Institute of Biomedical Sciences, University of São Paulo, São Paulo, Brazil \\ d Department and Institute of Psychiatry, University of São Paulo, São Paulo, Brazil \\ e Collegiate of Biological Sciences, Federal University of Vale do São Francisco, Petrolina, Brazil \\ ${ }^{\mathrm{f}}$ Department of Clinical Medicine and Translational Psychiatry Research Group, Faculty of Medicine, Federal University of Ceará, Fortaleza, CE, Brazil \\ ${ }^{\mathrm{g}}$ Service of Interdisciplinary Neuromodulation, Laboratory of Neurosciences (LIM-27), Institute of Psychiatry, São Paulo, Brazil
}

\section{A R T I C L E I N F O}

\section{Keywords}

Major depressive disorder

Heart rate variability

Vagal activity

Nonverbal behavior

Ethogram

Psychiatry

\begin{abstract}
A B S T R A C T
Background: Major depressive disorder (MDD) is associated with impairments in nonverbal behaviors (NVBs) and vagal activity. The polyvagal theory proposes that vagal activity regulates heart rate and NVBs by modulating a common anatomically and neurophysiologically discrete social engagement system. However, the association between these putative endophenotypes has not yet been explored. We hypothesize that in MDD, NVBs indicating positive affects and social interest and those indicating negative feelings and social disinterest could be associated with different patterns of vagal activity.

Methods: For this cross-sectional study we recruited 50 antidepressant-free participants with moderate-tosevere MDD. Vagal activity was indexed by heart rate variability (HRV) measures, and positive and negative nonverbal behaviors (NVBs) by a validated ethogram. Associations between NVBs and HRV were explored by bivariate analyses and multivariable models were adjusted by age, gender, depression severity, and self-reported positive and negative affects.

Results: HRV measures indicative of higher vagal activity were positively correlated with positive NVBs exhibited during the clinical interview. Conversely, NVBs related to negative affects, low energy and social disinterest were not associated with HRV.

Limitations: Absence of a control group.

Conclusions: The findings highlight that the examined depression endophenotypes (nonverbal behaviors and vagal activity) are related, shedding light on MDD pathophysiology in the context of the polyvagal theory.
\end{abstract}

\section{Introduction}

Several studies have investigated the role of nonverbal behaviors (NVBs) in social functioning in major depressive disorder (MDD). For instance, decreased facial and head movements are related to depression severity (Fiquer et al., 2013; Girard et al., 2014), and behavioral patterns can aid in differentiating depression from other psychiatric disorders in patients (Annen et al., 2012). Depression improvement is also associated with an increase in social interest behaviors (Fiquer

\section{et al., 2013; Girard et al., 2014).}

NVBs are controlled not only by the central, but also by the autonomic nervous system (ANS) (Appelhans and Luecken, 2008). According to the polyvagal theory (Porges, 2011), vagal motor pathways regulating heart rate and head striated muscles are anatomically and neurophysiologically part of the same system that induces complex adaptive and emotional responses. Consequently, the social engagement system, which regulates social behavior and communication, is linked to the myelinated vagus, and can only function efficiently when

Abbreviations: MDD, major depressive disorder; NVB, nonverbal behavior; HRV, heart rate variability; HF, high frequency; LF, low frequency; HDRS, Hamilton Depression Rating Scale; RMSSD, root mean squares of successive differences; PANAS, Positive and Negative Affect Schedule

* Correspondence to: Interdisciplinary Center for Applied Neuromodulation, Av. Professor Lineu Prestes, 2565, 3o andar, CEP 05508-000 São Paulo (SP), Brazil.

E-mail address: brunowsky@gmail.com (A.R. Brunoni). 
defensive circuits (such as the "fight or flight" response) are inhibited (Porges, 2011).

Hence, the inhibitory role of cortical structures is critical for downregulating vagal activity (Thayer et al., 2009). This might explain why disorders associated with prefrontal dysfunction, such as MDD, are associated with a reduction of vagal activity reflected by decreased heart rate variability (HRV) (Kemp et al., 2014). In fact, HRV is determined by a variety of physiological factors, particularly the ANS. In the resting state, the heart is under tonic inhibitory control by the parasympathetic (vagal) nervous system, whereas heart rate increases during increased sympathetic input on the sinoatrial node. (Kikuchi et al., 2009). In this context we refer to HRV as surrogate marker of ANS activity.

However, although patients with MDD present alterations in NVBs and vagal activity, the relationship between these has not yet been explored - and consequently was the aim of the present study. Our a priori hypothesis was that "positive" behaviors (i.e., nonverbal indicators of social interest, positive feelings and/or willingness to communicate) would be associated with higher vagal activity, whereas "negative" behaviors (i.e., nonverbal indicators of social disinterest, low energy and/or negative feelings) would be associated with lower vagal activity. This investigation may provide a better understanding of the role of the ANS in the pathophysiology of MDD in the context of polyvagal theory.

\section{Methods}

\subsection{Study design}

This cross-sectional study used ECG recordings and a previously validated ethogram, respectively, to evaluate HRV and NVBs. Patients were recruited from an ongoing clinical trial (Brunoni et al., 2015). The local and national Ethics Committee approved this study and all participants provided written informed consent. This study is in accordance with a recently published guideline on reporting psychiatric studies using HRV (Quintana et al., 2016).

\subsection{Subjects}

Participants were recruited by media advertisements and physician referrals. They were pre-screened in brief telephone and e-mail interviews, and those meeting the general criteria underwent additional onsite screening (Supplementary material).

\subsection{Procedure}

Prior to randomization or any intervention, HRV and NVBs were assessed. In most cases, HRV was collected immediately after the NVB assessment, although in some cases HRV was only collected the next day for logistical reasons.

\subsubsection{Heart rate variability (HRV)}

HRV is an ECG-based technique characterized by the examination of periodic and non-periodic oscillations of heartbeats which can be detected by changes in the length of the R-R intervals. Spectral analysis of the ECG distinguishes high (HF) and low (LF) frequency bands. HF is mainly associated with vagal activity, and LF with parasympathetic and sympathetic activity. Another HRV index is the root mean squares of successive differences (RMSSD), which is also associated with vagal activity. HRV procedures are described in the Supplementary Material.

\subsubsection{Behavioral assessment}

Behavioral assessment was performed during a 15-min interview by a trained psychologist (JTF). Patients were asked to describe their daily routine, current events, and mood state while being filmed. The camera was adjusted to record each patient's face and trunk. The footage was used to analyze patients' behavior, using ten $30 \mathrm{~s}$ sequences of time during the first $5 \mathrm{~min}$ of the interview. Two trained raters who were blinded to the study objectives recorded the presence (score 1) or absence (score 0) of 20 behavioral categories at each period of time, using a standardized ethogram (Fiquer et al., 2013) developed specifically to assess the behaviors patients exhibited during recorded clinical interviews. Raters performed at a uniform level of inter- and intra-rater reliability (kappa scores of 0.76 and 0.85 , respectively). From the 20 behavioral categories, 10 are associated with positive affects and/or favorable disposition to social contact ("positive behaviors"), and 10 are associated with negative affects, low energy and/or social disinterest ("negative behaviors").

\subsubsection{Clinical scales}

Patients were also evaluated by the Portuguese-validated versions of the Positive and Negative Affect Schedule (PANAS), State-Trait Anxiety Inventory (STAI) and HDRS-17 (Gorenstein and Andrade, 1996).

\subsection{Statistical analysis}

Analyses were conducted with Stata 12 software (Statacorp, College Station, TX, USA), and the sample was described by means and frequencies. The Shapiro-Wilk and Levene tests were used to check the normality and homoscedasticity of data, respectively. In all the analyses, results were significant at $\mathrm{p} \leq 0.05$.

Spearman's Rho test was used to evaluate associations between HRV variables, computed total scores of positive and negative NVBs, and continuous data relevant for our analyses such as age, depression severity (HDRS-17), duration of the current depressive episode, body mass index, number of previous depressive episodes, and scores from the Positive and Negative Affects Scale (PANAS) and the State-Trait Anxiety Inventory (STAI). T-tests were used to explore the influence of categorical variables such as gender, physical activity, social drinking, smoking status, hypertension, and use of benzodiazepines on HRV and NVB. Physical activity was assessed using the IPAQ questionnaire (Craig et al., 2003) and was categorized as inactive vs. active. "Social drinking" was defined as a drinking habit in social gatherings without the intention to get drunk or other patterns related to excessive alcohol use (binge drinking, heavy drinking). Importantly, participants presenting diagnoses of alcohol abuse or alcohol dependence were not included according to our eligibility criteria.

Univariate linear regression models were initially performed to further investigate the association between HRV and NVBs. The first step was to perform a natural logarithmic transformation of HRV measures to ensure data normalization. Next, HRV variables were introduced as outcome variables in our model and NVBs as predictors.

Subsequently we constructed multivariate linear regression models. Here we used a forced-entry method in which covariates were introduced based on clinical reasoning rather than testing the collected covariates. We did this because of the high number of available variables (since multiple testing could inflate type I error) and the relatively low number of observations $(n=50)$, which meant that introducing several predictors would lead to underpowered analyses, increasing type II error. The covariates age and gender were selected for their high influence on HRV measures and baseline depression and PANAS because of its possible influence on NVB measures. Finally, anxiety-trait and anxiety-state were assessed due to their influence on both HRV and NVB measures.

\section{Results}

Participants were recruited between February and July 2014. During this period, 130 volunteers were interviewed and 80 were excluded due to: depression of low/mild intensity $(n=28)$, use of 
Table 1

Demographic, clinical and behavioral characteristics of the sample.

\begin{tabular}{ll}
\hline Demographic and clinical variables & \\
Gender (Men/women) & $18 / 32$ \\
Active smokers, n (\%) & $7(14)$ \\
Social drinker, n (\%) & $16(32)$ \\
Physically inactive, n (\%) & $17(34)$ \\
Use of benzodiazepines, n (\%) & $22(44)$ \\
Obesity, n(\%) & $11(22)$ \\
Age, mean (SD) & $45.63(13.55)$ \\
HDRS-17 (M; \pm SD) & $21.66(3.76)$ \\
PA-PANAS, mean (SD) & $17.27(7.10)$ \\
NA-PANAS, mean (SD) & $27.02(8.48)$ \\
RMSSD (ms), mean(SD) (*) & $3.51(0.90)$ \\
HF (ms), mean (SD) (*) & $5.76(1.73)$ \\
LF (ms), mean (SD) (*) & $5.74(1.44)$ \\
STAI-S, mean (SD) & $53.23(11.65)$ \\
STAI-T, mean (SD) & $63.88(8.65)$ \\
BMI, mean (SD) & $26.07(5.6)$ \\
Dur of dep episode, mean(SD) & $21.07(17.31)$ \\
N of dep episodes, mean (SD) & $5.54(4.52)$
\end{tabular}

Nonverbal behavioral variables

Positive behaviors, mean (SD)

Lean forward

Head to Side

Head Up

Yes-nodding

Illustrative gestures

Eye contact

Laughter

Symmetric smile

Raised eyebrows

Verbal backchannel

Negative behaviors, mean (SD)

Shrug

Head down

No-nodding

Folded arms

Adaptative gestures

Assymmetric smile

Lips down

Tight lips

Frown

Cry reproduced the same findings from the univariate analysis, showing that positive NVBs were still associated with RMSSD and HF. Furthermore, in all models age was significantly and negatively associated with the HRV measures.

\section{Discussion}

According to our hypothesis, a direct correlation was observed between HF and RMSSD with positive NVBs - i.e., depressed patients with higher vagal activity displayed more pro-social and positive feeling behaviors. This finding was present regardless of patient age, gender, depression severity and PANAS and STAI scores.

Our finding supports evidence that NVBs can access automatic processes related to socio-emotional maladjustments and depressive states (Annen et al., 2012; Fiquer et al., 2013; Girard et al., 2014). As a result, these findings suggest the relevance of a multidisciplinary approach to depression to aid researchers in actively exploring behavioral and vagal activity symptoms in this disease, since they share common physiological mechanisms. For instance, decreased HRV observed in MDD (Kemp et al., 2010) is an important issue to be addressed when providing health care for these patients. Moreover, due to the close link between HRV and further appearance of depressive episodes (Jandackova et al., 2016), interventions that increase HRV could potentially be used to prevent depressive episodes.

Interestingly, LF was associated with positive behavior in the correlation analysis but not in the multiple regressions. Although LF is traditionally considered a marker of sympathetic modulation (Malliani et al., 1991), recent evidence has shown that it is influenced by both sympathetic and vagal influences (Reyes Del Paso et al., 2013). Therefore, LF may not be a specific enough biomarker to examine NVBs.

Contrary to our initial hypothesis, no association was found between measures of HRV and negative behaviors. This finding, however, is in accordance with the polyvagal theory which maintains that social behavior and communication, which are linked to the myelinated vagus, can only be expressed efficiently when the defensive circuits (non-myelinated vagus and sympathetic system) are inhibited (Porges, 2011). Depressed individuals might experience increased activation of the sympathetic system, which in turn decreases myelinated vagus activity, leading to social behavior and communication. Thus, negative behavior in MDD patients could be primarily associated with the sympathetic system, which is not very well assessed by HRV measures (Eckberg, 1997; Porges, 2011). To our knowledge, this is the first study to investigate associations between nonverbal behaviors and vagal activity, both putative endophenotypes of depression. Future studies with depressed patients could improve assessments of the sympathetic system using other appropriate methods, such as skin conductance, pupillary reflex, pre-ejection period and muscle sympathetic nerve activity (Verrier and Harper, 2011).

\section{Limitations}

Study limitations include the absence of a control group and the cross-sectional design. We also did not explore the previously described influence of suicidality on HRV (Wilson et al., 2016), since we excluded patients with suicidal ideation and did not collect data on lifetime suicidal attempts. Furthermore, although patients with hypertension did not use beta blockers, we did not evaluate the effects of antihypertensive medications that act on the renin-angiotensin-aldosterone system, which can influence HRV via modulation of the nucleus tractus solitarius (NTS) (Daubert et al., 2012).

\section{Conclusions}

Vagal activity and nonverbal positive social engagement, two putative endophenotypes of MDD, might be related. The cortical/ 

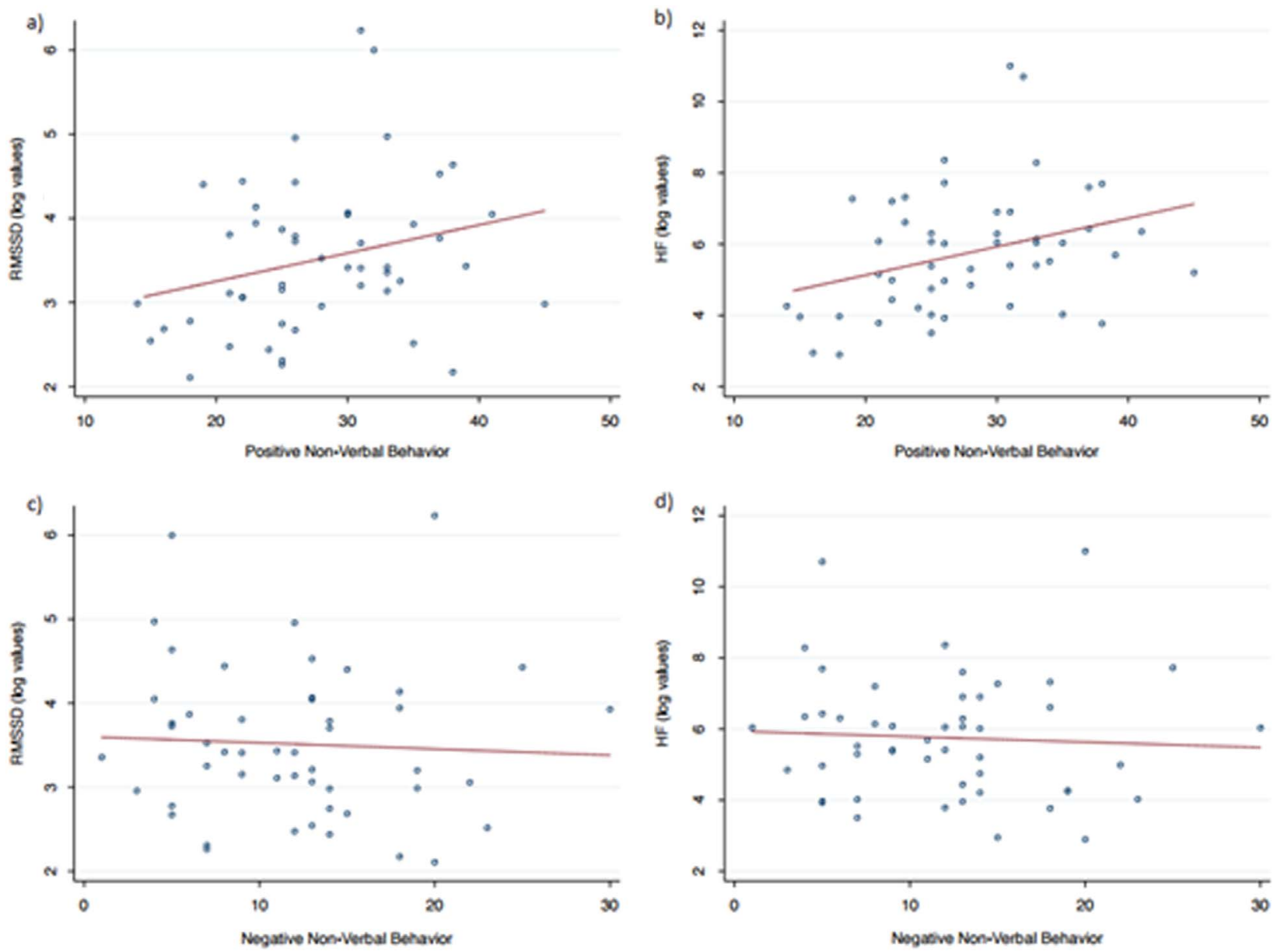

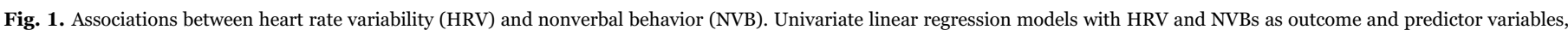

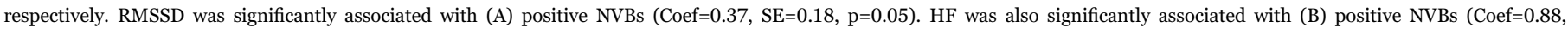

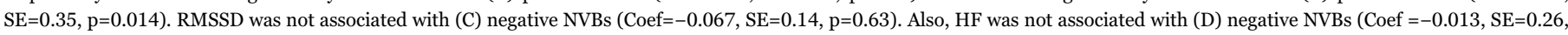
$\mathrm{p}=0.62$ ). Finally, LF was associated with neither positive nor negative NVBs (Coef $=0.49, \mathrm{SE}=0.3, \mathrm{p}=0.1$ and $\mathrm{Coef}=0.1, \mathrm{SE}=0.22$, $\mathrm{p}=0.64$ ).

subcortical dysfunction observed in MDD (i.e., decreased prefrontal cortical activity and increased activity in the amygdala and other subcortical structures) may explain this finding, and should be explored further by studies using other biomarkers (e.g., ANS peripheral markers such as pupillary reflex, skin conductance, pre-ejection period and muscle sympathetic nerve activity). This study shed light on the pathophysiology of MDD and is consistent with the polyvagal theory in MDD.

\section{Acknowledgements}

RAF is recipient of a FAPESP (Sao Paulo Research Foundation) "Scientific Initiation" fellowship (14/20799-6). ARB is supported by the following Grants: 2013 NARSAD Young Investigator from the Brain \& Behavior Research Foundation (Grant number 20493), 2013 FAPESP Young Researcher from the São Paulo State Foundation (Grant number 20911-5) and National Council for Scientific and Technological Development (CNPq, Grant number 470904). ARB is also recipient of a research fellowship award from CNPq (303197). AFC is supported by a research fellowship award from CNPq (Level 2). JTF and CG were also supported by the São Paulo Research Foundation (FAPESP), process number 11/51732-6 and 12/50879-6, respectively.

\section{Appendix A. Supporting information}

Supplementary data associated with this article can be found in the online version at doi:10.1016/j.jad.2016.11.010.

\section{References}

Annen, S., Roser, P., Brune, M., 2012. Nonverbal behavior during clinical interviews: similarities and dissimilarities among schizophrenia, mania, and depression. J. Nerv. Ment. Dis. 200, 26-32.

Appelhans, B.M., Luecken, L.J., 2008. Heart rate variability and pain: associations of two interrelated homeostatic processes. Biol. Psychol. 77, 174-182.

Brunoni, A.R., Sampaio-Junior, B., Moffa, A.H., Borrione, L., Nogueira, B.S., Aparicio, L.V., Veronezi, B., Moreno, M., Fernandes, R.A., Tavares, D., Bueno, P.V., Seibt, O., Bikson, M., Fraguas, R., Bensenor, I.M., 2015. The Escitalopram versus Electric Current Therapy for Treating Depression Clinical Study (ELECT-TDCS): rationale and study design of a non-inferiority, triple-arm, placebo-controlled clinical trial. Sao Paulo Med. J. 133, 252-263.

Craig, C.L., Marshall, A.L., Sjostrom, M., Bauman, A.E., Booth, M.L., Ainsworth, B.E., Pratt, M., Ekelund, U., Yngve, A., Sallis, J.F., Oja, P., 2003. International physical activity questionnaire: 12-country reliability and validity. Med. Sci. Sports Exerc. 35, 1381-1395.

Daubert, D.L., McCowan, M., Erdos, B., Scheuer, D.A., 2012. Nucleus of the solitary tract catecholaminergic neurons modulate the cardiovascular response to psychological stress in rats. J. Physiol. 590, 4881-4895.

Eckberg, D.L., 1997. Sympathovagal balance: a critical appraisal. Circulation 96, 3224-3232.

Fiquer, J.T., Boggio, P.S., Gorenstein, C., 2013. Talking bodies: nonverbal behavior in the assessment of depression severity. J. Affect Disord. 150, 1114-1119.

Girard, J.M., Cohn, J.F., Mahoor, M.H., Mavadati, S.M., Hammal, Z., Rosenwald, D.P., 2014. Nonverbal social withdrawal in depression: evidence from manual and automatic analysis. Image Vis. Comput 32, 641-647.

Gorenstein, C., Andrade, L., 1996. Validation of a portuguese version of the beck depression inventory and the state-trait anxiety inventory in brazilian subjects. Braz. J. Med. Biol. Res. 29, 453-457.

Jandackova, V.K., Britton, A., Malik, M., Steptoe, A., 2016. Heart rate variability and depressive symptoms: a cross-lagged analysis over a 10-year period in the Whitehall II study. Psychol. Med. 46, 2121-2131.

Kemp, A.H., Brunoni, A.R., Santos, I.S., Nunes, M.A., Dantas, E.M., Carvalho de Figueiredo, R., Pereira, A.C., Ribeiro, A.L., Mill, J.G., Andreao, R.V., Thayer, J.F., 
Bensenor, I.M., Lotufo, P.A., 2014. Effects of depression, anxiety, comorbidity, and antidepressants on resting-state heart rate and its variability: an ELSA-Brasil cohort baseline study. Am. J. Psychiatry.

Kemp, A.H., Quintana, D.S., Gray, M.A., Felmingham, K.L., Brown, K., Gatt, J., 2010. Impact of depression and antidepressant treatment on heart rate variability: a review and meta-analysis. Biol. Psychiatry 67, 1067-1074.

Kikuchi, M., Hanaoka, A., Kidani, T., Remijn, G.B., Minabe, Y., Munesue, T., Koshino, Y., 2009. Heart rate variability in drug-naive patients with panic disorder and major depressive disorder. Prog. Neuropsychopharmacol. Biol. Psychiatry 33, 1474-1478.

Malliani, A., Pagani, M., Lombardi, F., Cerutti, S., 1991. Cardiovascular neural regulation explored in the frequency domain. Circulation 84, 482-492.

Porges, S.W., 2011. The Polyvagal Theory: neurophysiological Foundations of Emotions, Attachment, Communication, and Self-regulation (Norton Series on Interpersonal Neurobiology). WW Norton \& Company, New York.

Quintana, D.S., Alvares, G.A., Heathers, J.A., 2016. Guidelines for Reporting articles on psychiatry and heart rate variability (GRAPH): recommendations to advance research communication. Transl. Psychiatry 6, e803.

Reyes Del Paso, G.A., Langewitz, W., Mulder, L.J., van Roon, A., Duschek, S., 2013. The utility of low frequency heart rate variability as an index of sympathetic cardiac tone: a review with emphasis on a reanalysis of previous studies. Psychophysiology.

Thayer, J.F., Hansen, A.L., Saus-Rose, E., Johnsen, B.H., 2009. Heart rate variability, prefrontal neural function, and cognitive performance: the neurovisceral integration perspective on self-regulation, adaptation, and health. Ann. Behav. Med. 37, 141-153.

Verrier, R.L., Harper, R.M., 2011. Cardiovascular physiology: central and autonomic regulation. In: Kryger, M.H., Roth, T., Dement, W.C. (Eds.), Principles and Practice of Sleep Medicine. Elsevier, St. Louis, Missouri.

Wilson, S.T., Chesin, M., Fertuck, E., Keilp, J., Brodsky, B., Mann, J.J., Sonmez, C.C., Benjamin-Phillips, C., Stanley, B., 2016. Heart rate variability and suicidal behavior Psychiatry Res. 240, 241-247. 